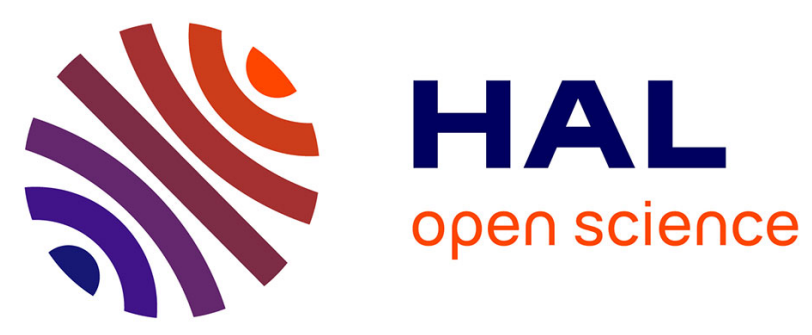

\title{
PROTOSOLAR CARBON ISOTOPIC COMPOSITION: IMPLICATIONS FOR THE ORIGIN OF METEORITIC ORGANICS
}

Ko Hashizume, Marc Chaussidon, Bernard Marty, Kentaro Terada

\section{- To cite this version:}

Ko Hashizume, Marc Chaussidon, Bernard Marty, Kentaro Terada. PROTOSOLAR CARBON ISOTOPIC COMPOSITION: IMPLICATIONS FOR THE ORIGIN OF METEORITIC ORGANICS.

The Astrophysical Journal, 2003, 600, pp.480-484. insu-01882002

\section{HAL Id: insu-01882002 \\ https://hal-insu.archives-ouvertes.fr/insu-01882002}

Submitted on 26 Sep 2018

HAL is a multi-disciplinary open access archive for the deposit and dissemination of scientific research documents, whether they are published or not. The documents may come from teaching and research institutions in France or abroad, or from public or private research centers.
L'archive ouverte pluridisciplinaire $\mathbf{H A L}$, est destinée au dépôt et à la diffusion de documents scientifiques de niveau recherche, publiés ou non, émanant des établissements d'enseignement et de recherche français ou étrangers, des laboratoires publics ou privés. 
The Astrophysical Journal, 600:480-484, 2004 January 1

(C) 2004. The American Astronomical Society. All rights reserved. Printed in U.S.A.

\title{
PROTOSOLAR CARBON ISOTOPIC COMPOSITION: IMPLICATIONS FOR THE ORIGIN OF METEORITIC ORGANICS
}

\author{
Ko Hashizume \\ Department of Earth and Space Sciences, Graduate School of Science, Osaka University, Toyonaka, Osaka 560-0043, Japan; kohash@ess.sci.osaka-u.ac.jp \\ Marc Chaussidon and Bernard Marty ${ }^{1}$ \\ Centre de Recherches Pétrographiques et Géochimiques, CNRS, BP 20, 54501 Vandoeuvre-lès-Nancy Cedex, France
}

AND

Kentaro Terada

Department of Earth and Planetary Sciences, Hiroshima University, Higashi-Hiroshima 739, Japan

Received 2003 May 21; accepted 2003 September 4

\begin{abstract}
New ion probe isotopic measurements of carbon trapped within the $50 \mathrm{~nm}$ thick surface layer of lunar regolith grains strongly suggest that solar wind $\mathrm{C}$ is depleted in ${ }^{13} \mathrm{C}$ by at least $10 \%$ relative to terrestrial $\mathrm{C}$. In order to account for the general ${ }^{13} \mathrm{C}$ enrichment of planetary $\mathrm{C}$ relative to solar $\mathrm{C}$, we propose that the main carriers of $\mathrm{C}$ in these objects, i.e., organics, were formed in an environment that allowed a strong isotopic enrichment of ${ }^{13} \mathrm{C}$ in the solid phase. Such an environment is most likely a dense and warm circumstellar or interstellar gas medium, which could well correspond to the nebula surrounding the proto-Sun, where isotopic fractionation could be triggered by photochemical reactions.
\end{abstract}

Subject headings: astrochemistry — ISM: abundances — Moon — planetary systems: protoplanetary disks — solar wind - ultraviolet: solar system

\section{INTRODUCTION}

Knowledge of the carbon isotopic composition of the Sun, and thus by inference that of the solar nebula, is essential to understanding the origin and evolution of organics in the nascent solar system, especially their primary reaction pathways. Organics are indeed the dominant form of $\mathrm{C}$ in primitive planetary objects, such as primitive meteorites (Sephton \& Gilmour 2000; Alexander et al. 1998), and they are believed to be the main suppliers of $\mathrm{C}$ to planetary bodies. During the process of organic formation, the conversion of carbon monoxide to more reactive forms of $\mathrm{C}$-bearing gas can lead to large C isotopic fractionation (Smith \& Adams 1980; van Dishoeck \& Black 1988; Yuen et al. 1990). The H and N isotopic compositions of terrestrial planets and meteorites are drastically different from their protosolar bulk compositions (Robert et al. 1987; Hashizume et al. 2000), consistent with the extensive isotopic fractionation expected to occur during low-temperature reactions in the interstellar or circumstellar gas medium (Tielens 1997; Terzieva \& Herbst 2000). However, because of the existence of competitive chemical pathways inducing $\mathrm{C}$ isotopic fractionation along opposite directions (van Dishoeck \& Black 1988; Warin, Benayoun, \& Viala 1996), the overall degree of $C$ isotopic fractionation through organic matter synthesis is difficult to predict.

This study is a continuation of our previous work on the $\mathrm{N}$ isotopic composition of the solar wind (SW; Hashizume et al. 2000). By measuring $\mathrm{N}$ isotopic variations within the first $50 \mathrm{~nm}$ thick layers of minerals of the lunar regolith, it was possible to identify implanted SW N and to constrain its N isotopic composition. This experiment led us to propose that

\footnotetext{
${ }^{1}$ École Nationale Supérieure de Géologie, 54501 Vandoeuvre-lès-Nancy Cedex, France.
}

$\mathrm{N}$ in planetary organics is systematically enriched in ${ }^{15} \mathrm{~N}$, compared to the solar or protosolar composition (Hashizume et al. 2000). This conclusion, that the solar ${ }^{15} \mathrm{~N} /{ }^{14} \mathrm{~N}$ ratio is lower than the terrestrial ratio by at least $24 \%$, was further confirmed by the Galileo probe measurement of the Jovian atmosphere (Owen et al. 2001).

We have measured by ion microprobe the depth profile of the carbon isotopic composition in mineral grains from lunar regolith sample 79035. This sample is enriched in SW rare gases and is thought to have been exposed to the SW at about 1 Gyr ago (Wieler et al. 1996). In addition, it contains at its surface solar-like (deuterium-free) hydrogen and $\left({ }^{15} \mathrm{~N}\right.$-poor) nitrogen, whereas the amount of (D- and ${ }^{15} \mathrm{~N}$-rich) planetary volatiles coming from micrometeorites bombarded to the lunar surface seems to be relatively minor (Hashizume et al. 2000; Hashizume, Marty, \& Wieler 2002). Previous bulk measurements of carbon isotopes in this sample showed a slight depletion of ${ }^{13} \mathrm{C}$ relative to the terrestrial standard ${ }^{13} \mathrm{C} /{ }^{12} \mathrm{C}$ ratio of $\sim 1 / 89$, with $\delta^{13} \mathrm{C}$ values of $-7 \%$ or $-13 \%$ (Becker \& Epstein 1981), where $\delta^{13} \mathrm{C}$ denotes the permil deviation of ${ }^{13} \mathrm{C} /{ }^{12} \mathrm{C}$ from the terrestrial standard ratio. The bulk $\delta^{13} \mathrm{C}$ value of this sample is close to the lower limit of the $\delta^{13} \mathrm{C}$ range between $-20 \%$ and $+25 \%$ observed among various lunar samples (Becker 1980), suggesting that a ${ }^{13} \mathrm{C}$-poor component is present in this sample.

\section{METHODS}

Ion probe analyses were performed at the Centre de Recherches Pétrographiques et Géochimiques (CRPG)-CNRS (Nancy, France), using the Cameca ims-1270 (Hashizume et al. 2000). Relatively flat surfaces of nonpolished mineral grains coated with gold and embedded in indium were sputtered by a $0.5-1.5 \mathrm{nA}$ primary $\mathrm{Cs}^{+}$beam using electron flooding. Secondary ions of $\mathrm{H}^{-}$and $\mathrm{C}^{-}$were measured using an electron 
multiplier. The $\mathrm{H}$ and $\mathrm{C}$ isotopes were measured in monocollection and multicollection, respectively. $\mathrm{C}$ and $\mathrm{H}$ analyses of the same grain were performed independently at different sites. A mass resolution power $(M / \Delta M)$ of greater than 3000 was required to resolve ${ }^{12} \mathrm{CH}^{-}$from ${ }^{13} \mathrm{C}^{-}$. We used a midocean

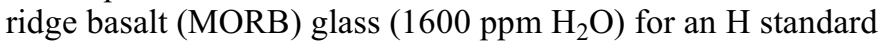
and a synthesized $\mathrm{C}$-bearing silicate glass for a $\mathrm{C}$ standard $(\sim 6000 \mathrm{ppm} \mathrm{C})$. The standard mean deviation of the ${ }^{13} \mathrm{C} /{ }^{12} \mathrm{C}$ ratio for the standard glass, comparing the ratios obtained at each $5 \mathrm{~nm}$ depth (which corresponds to the depth resolution for the $\mathrm{C}$ measurements described in Fig. 1) was about 7\%, which appears to be limited by the statistical counting error of the ${ }^{13} \mathrm{C}^{-}$ ion. Deviations of the average ${ }^{13} \mathrm{C} /{ }^{12} \mathrm{C}$ ratios among independent standard measurements within the same day were about $1 \%-5 \%$, depending on the day. The average values varied by $\pm 6 \%$ day by day. The average instrumental mass discrimination for the ${ }^{13} \mathrm{C} /{ }^{12} \mathrm{C}$ ratio was $-25 \%$. The upper limit for the blank $\mathrm{C}$ is estimated from the count rate of $\mathrm{C}^{-}$ detected at the very surface $(<5 \mathrm{~nm})$ of the sample grain, which perhaps represents the sum of contamination $\mathrm{C}$ in the coating gold and the analytical blank, such as the outgassing of the sample chamber. In grain $02 \mathrm{~B}$, for example, the count rate of ${ }^{12} \mathrm{C}^{-}$at the surface corresponded to a $\mathrm{C}$ concentration of $200 \mathrm{ppm}$ in silicate samples, which was $\sim 2 \%$ of the maximum count rate detected at the grain interior. The isotopic ratio of the blank $\mathrm{C}\left(\delta^{13} \mathrm{C}=-6 \% \pm 24 \%\right.$ ) was essentially the same as what is classical for terrestrial contaminants. The contribution of blank $\mathrm{C}$ to the total $\mathrm{C}$ signal is thus quite negligible, so no blank subtractions are performed for the data in this study. The $\delta \mathrm{D}$ and $\delta^{13} \mathrm{C}$ stand, respectively, for the permil deviations of the $\mathrm{D} / \mathrm{H}$ ratio relative to standard mean oceanic water $\left(\mathrm{D} / \mathrm{H}=1.56 \times 10^{-4}\right)$ and the ${ }^{13} \mathrm{C} /{ }^{12} \mathrm{C}$ ratio relative to terrestrial standard calcite (Pee Dee Belemnite; ${ }^{13} \mathrm{C} /{ }^{12} \mathrm{C}=$ 0.0112).

\section{ISOTOPIC MEASUREMENT OF SOLAR CARBON IN LUNAR REGOLITH GRAINS}

The results show large variations of the $\mathrm{C}$ isotopic composition with depth from the grain surface to its interior (Fig. 1). The $\delta^{13} \mathrm{C}$ values decreased (Fig. 1a) abruptly from values close to $0 \%$ at the surface to values down to $-105 \%$ o $\pm 20 \%$, observed on two independent measurements at depths of 16 or $28 \mathrm{~nm}$, and then increased up to $+10 \%$ at depths of $>100 \mathrm{~nm}$. The C concentration (Fig. $1 b$ ) reached its maximum (7000-8000 ppm) at depths of 50$100 \mathrm{~nm}$ and regularly decreased at deeper parts, suggesting that $\mathrm{C}$ is a surface-correlated component. The $\mathrm{C}$ component present in trace amounts at the very surface of the grains is most likely a terrestrial contaminant. However, $\mathrm{C}$ found at more than $100 \mathrm{~nm}$ with near-zero $\delta^{13} \mathrm{C}$ values must be indigenous to the grains, because of its much higher concentration. The similarity of the $\delta^{13} \mathrm{C}$ profiles observed in several grains (Table 1) demonstrates the existence of two trapped components within the $200 \mathrm{~nm}$ thick surface layer of the grains, a ${ }^{13} \mathrm{C}$-poor component with a $\delta^{13} \mathrm{C}$ value lower than $-105 \%$ and a component implanted at greater depths, with higher $\delta^{13} \mathrm{C}$ values that range between $-30 \%$ and $+10 \%$ depending on grains. Assuming a simple binary mixing of these two components, it is possible to compute the depth distribution of the two components (Fig. 1c). The ${ }^{13} \mathrm{C}$-poor component reaches a maximum concentration at depths of typically $50-70 \mathrm{~nm}$, whereas the second component reaches a concentration plateau at greater depths of $100-150 \mathrm{~nm}$.

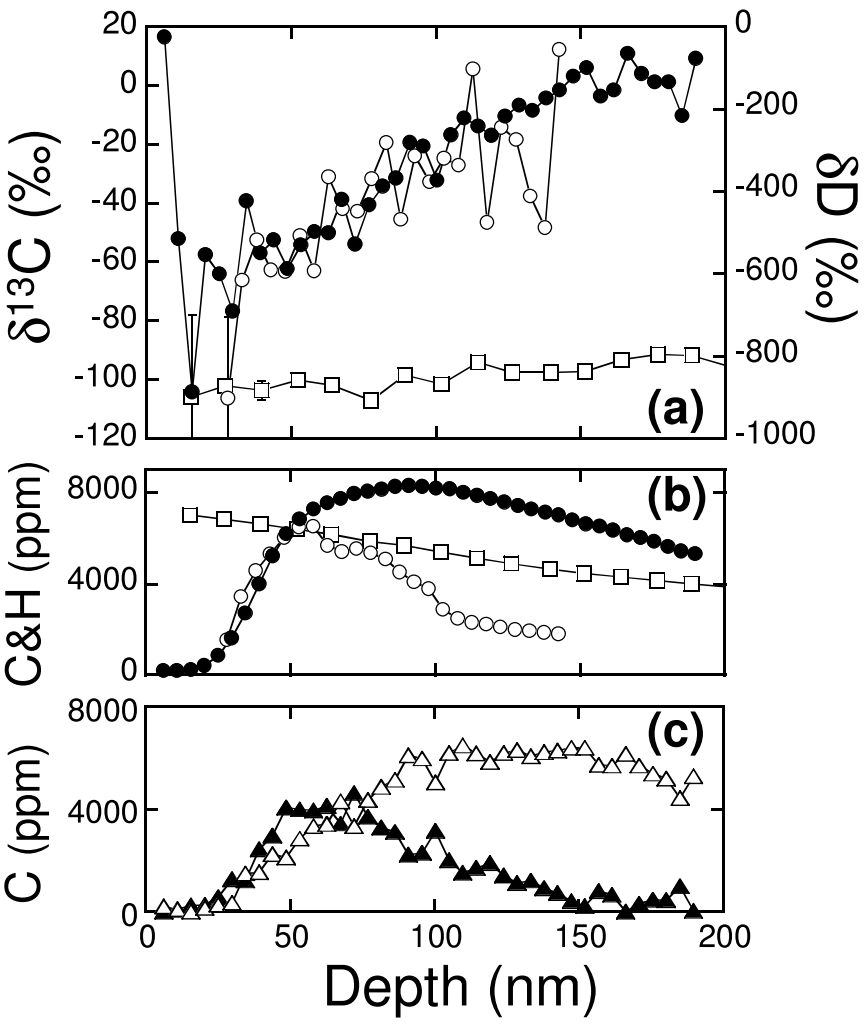

FIg. 1.-Example of depth profiles of isotopic compositions and concentrations of $\mathrm{C}$ and $\mathrm{H}$ in pyroxene grain $02 \mathrm{~B}$ from lunar regolith 79035. (a) The $\delta^{13} \mathrm{C}$ values are plotted as circles and the $\delta \mathrm{D}$ values as squares. $\mathrm{C}$ analyses are performed twice: first ( filled circles) by rastering the primary beam over $50 \mu \mathrm{m} \times 50 \mu \mathrm{m}$ and second (open circles) in nonrastering mode with a $\sim 30 \mu \mathrm{m}$ spot. The sputtering rates of the samples range from $0.07 \mathrm{~nm} \mathrm{~s}^{-1} \mathrm{nA}^{-1}$ in rastering mode to $0.19 \mathrm{~nm} \mathrm{~s}^{-1} \mathrm{nA}^{-1}$ in static mode. The $1 \sigma$ error bars are attached only to a representative value for each analysis, and others are omitted. (b) $\mathrm{C}$ and $\mathrm{H}$ concentrations, plotted with the same symbols as in $(a)$. The difference between the two $\mathrm{C}$ concentration profiles could be due to uncertainties in the estimation either of the sputtering rates, primary beam intensities, emissivities of secondary $\mathrm{C}^{-}$ions due to a geometry effect that may occur by measuring different locations of an unpolished grain, or combination of these factors. (c) Concentrations of two isotopically distinct $\mathrm{C}$ components for the first $\mathrm{C}$ analysis, assuming that the observed carbon profile results from a binary mixture of a ${ }^{13} \mathrm{C}$-poor component $\left(\delta^{13} \mathrm{C} \leq-105 \%\right.$; filled triangles $)$ and a ${ }^{13} \mathrm{C}$-rich component $\left(\delta^{13} \mathrm{C} \leq 10 \%\right.$; open triangles).

The $\delta \mathrm{D}$ value down to $-900 \%$ ( $\left.\mathrm{D} / \mathrm{H} \sim 1.6 \times 10^{-5}\right)$ (Fig. $1 a$ ) confirms that it contains a substantial amount of the solar component, with little, if any, contribution from meteoritic $\mathrm{H}$ that normally shows near-zero or positive $\delta \mathrm{D}$ values (Robert \& Epstein 1982; Robert et al. 1987). Among the different grains, the concentrations of the two trapped $\mathrm{C}$ components show a positive correlation with those of solar H (Fig. 2). Although this correlation strongly suggests that these surface-correlated $\mathrm{C}$ components are derived from the Sun, the observed $\mathrm{H} / \mathrm{C}$ abundance ratio (3-18 atoms per atom) is much lower than the abundance ratio measured in the solar photosphere $(2800$ atoms per atom; Anders \& Grevesse 1989), evidencing H loss from lunar soil grains by diffusion (Li \& Wittenberg 1992), otherwise attested to by the smoothness of the solar $\mathrm{H}$ concentration profile (Fig. 1b). Such $\mathrm{H}$ loss does not preclude the use of this element to monitor the dose of solar irradiation. In fact, the $\mathrm{H}$ concentration of lunar soils is correlated with the maturity index $\left(I_{S} / \mathrm{FeO}\right.$, which represents the fraction of iron oxide reduced to metallic iron by implantation of solar protons; Bustin \& Gibson 1992), 
TABLE 1

Summary of C and H Isotope Measurements on Grains from Lunar Regolith 79035

\begin{tabular}{|c|c|c|c|c|c|c|c|c|}
\hline \multirow[b]{2}{*}{ GRAIN } & \multirow[b]{2}{*}{ MineRAL } & \multirow[b]{2}{*}{$\begin{array}{l}\text { DePTH C MEAS. } \\
(\mathrm{nm})\end{array}$} & \multicolumn{3}{|c|}{ CONCENTRATION } & \multicolumn{3}{|c|}{$\delta^{13} \mathrm{C}$} \\
\hline & & & $\begin{array}{c}\text { Solar H } \\
(\mathrm{ppm})\end{array}$ & $\begin{array}{c}{ }^{13} \mathrm{C} \text {-poor C } \\
(\mathrm{ppm})\end{array}$ & $\begin{array}{c}{ }^{13} \mathrm{C} \text {-rich C } \\
(\mathrm{ppm})\end{array}$ & $\begin{array}{l}\text { Lowest } \\
(\%)\end{array}$ & $\begin{array}{c}\text { Highest } \\
(\%)\end{array}$ & $\begin{array}{c}{ }^{13} \mathrm{C} \text {-rich } \\
(\% 0)\end{array}$ \\
\hline $02 \mathrm{~A} \ldots \ldots . .$. & Pyroxene & 150 & 3489 & 3021 & 4431 & -63 & -34 & $-10^{*}$ \\
\hline $02 \mathrm{~B} \ldots \ldots \ldots$ & Pyroxene & 190 & 3304 & 2167 & 4277 & -106 & +11 & +10 \\
\hline $02 \mathrm{C} \ldots \ldots . .$. & Pyroxene & 190 & 2859 & 3966 & 10570 & -60 & -30 & -30 \\
\hline $02 \mathrm{D} \ldots \ldots \ldots$ & Pyroxene & 200 & 1169 & 1406 & 2492 & -74 & -18 & -15 \\
\hline $02 \mathrm{E} \ldots \ldots \ldots$ & Pyroxene & 120 & 923 & 1920 & 2565 & -63 & -43 & $-10^{*}$ \\
\hline $02 \mathrm{~F} \ldots \ldots \ldots$ & Ilmenite & 140 & 319 & 488 & 563 & -84 & -33 & $-10^{*}$ \\
\hline
\end{tabular}

Notes.-The concentrations given here correspond to micrograms of $\mathrm{C}$ or $\mathrm{H}$ components cumulated over given depths divided by the mass of the sputtered volumes. The cumulating depths for ${ }^{13} \mathrm{C}$-poor $\mathrm{C},{ }^{13} \mathrm{C}$-rich $\mathrm{C}$, and solar $\mathrm{H}$ components are 120, 200 (or the maximum measured depth indicated in the table), and $400 \mathrm{~nm}$, respectively, which correspond to the depths at which the respective components are abundantly observed. Concentrations of solar $\mathrm{H}$ were calculated assuming that the measured $\mathrm{H}$ is composed of a binary mixture between a solar component with no $\mathrm{D}$ and a normal (terrestrial) component with a $\delta \mathrm{D}$ value of $-100 \%$. The total measured carbon is assumed to be the result of mixing between a ${ }^{13} \mathrm{C}$-poor component and a ${ }^{13} \mathrm{C}$-rich component. The ${ }^{13} \mathrm{C}$-poor component is assumed to possess a $\delta^{13} \mathrm{C}$ value of $-105 \%$, whereas the $\delta^{13} \mathrm{C}$ values of the ${ }^{13} \mathrm{C}$-rich component (described in the column labeled "13 $\mathrm{C}$-rich") are assumed to be variable among grains, corresponding to the highest values observed at the deepest part, i.e., at $\sim 200$ $\mathrm{nm}$ depth. However, a moderate $\delta^{13} \mathrm{C}$ value of $-10 \%$ for the ${ }^{13} \mathrm{C}$-rich component was assumed for grains $02 \mathrm{~A}, 02 \mathrm{E}$, and $02 \mathrm{~F}$, whose measurements terminated at shallower depth. Contributions of ${ }^{13} \mathrm{C}$-rich $\mathrm{C}$ at the unmeasured parts of these grains to their average concentrations are estimated from the profiles of other grains to be less than $10 \%$.

indicating that the fraction of $\mathrm{H}$ loss is rather constant within the regolith.

The ${ }^{13} \mathrm{C}$-poor carbon component, abundant within a depth interval from 30 to $70 \mathrm{~nm}$ (Fig. 1c), is most likely implanted SW C, because this depth interval is the one expected for implantation into silicates of ions having kinetic energies characteristic of the $\mathrm{SW}\left(\sim 1 \mathrm{keV}\right.$ nucleon ${ }^{-1}$; Ziegler, Biersack, \& Littmark 1985). By analogy with the depth distribution observed for solar rare gases in lunar soil grains (Wieler 1998), the second trapped C component, which reaches a maximum concentration at depths $\gtrsim 100 \mathrm{~nm}$ (Fig. 1c) possibly corresponds to solar energetic particles (SEPs), which have higher kinetic energies. Although isotopic and elemental abundance ratios of SEPs are known to be highly variable from flare to flare, the long-term averaged isotopic compositions, such as those recorded in lunar and meteoritic samples, are constant and systematically enriched in heavy isotopes relative to SW. A comparison of the isotopic composition of SEPs and SW rare gases suggests that the enrichment factor is roughly proportional to the square of the mass (Wieler 1998). Applying this empirical law to carbon, $\delta^{13} \mathrm{C}_{\mathrm{SEP}}$ is expected to be higher by $170 \%$ compared to $\delta^{13} \mathrm{C}_{\mathrm{SW}}$. The observed difference in the $\delta^{13} \mathrm{C}$ values between the light and heavy components $(\sim 120 \%)$ agrees reasonably well with this expectation, especially if one considers that the $\delta^{13} \mathrm{C}$ value of the pure SW end member might be somewhat lower than $-105 \%$ o $\pm 20 \%$ because of possible unresolved minor contributions of the SEP component and/or the surface contamination. The apparent overabundance of SEP $\mathrm{C}$ relative to $\mathrm{SW} C\left(\mathrm{C}_{\mathrm{SEP}} / \mathrm{C}_{\mathrm{SW}} \sim 2\right)$, compared to the extremely small fraction of high-energy solar ions in space (e.g., $10^{-4}$ to $\sim 10^{-5}$ for $>0.1 \mathrm{MeV}$ nucleon ${ }^{-1}$ particles; Wieler 1998), is also consistent with what has been observed for rare gases (Wieler 1998).

The $\mathrm{C}$ isotopic composition that we estimate for SW implanted in the lunar regolith is likely to be close to that of the solar photosphere and the bulk solar isotopic ratio. First, a large degree of isotopic fractionation of the SW upon implantation due to a difference in stopping depths between ${ }^{12} \mathrm{C}$ and ${ }^{13} \mathrm{C}$ is unlikely to occur. This is because ion implantation simulations (Ziegler et al. 1985) suggest that implantation depths are dependent on velocities rather than on the total kinetic energy ( $\propto$ mass) of SW ions, while their bulk velocities are observed to be fairly constant among various heavy ions (Bochsler 1989). Second, it is also unlikely to expect extensive $\mathrm{C}$ isotopic fractionation during ion acceleration processes at the source of the SW, because elemental abundance ratios of SW ions with similar first

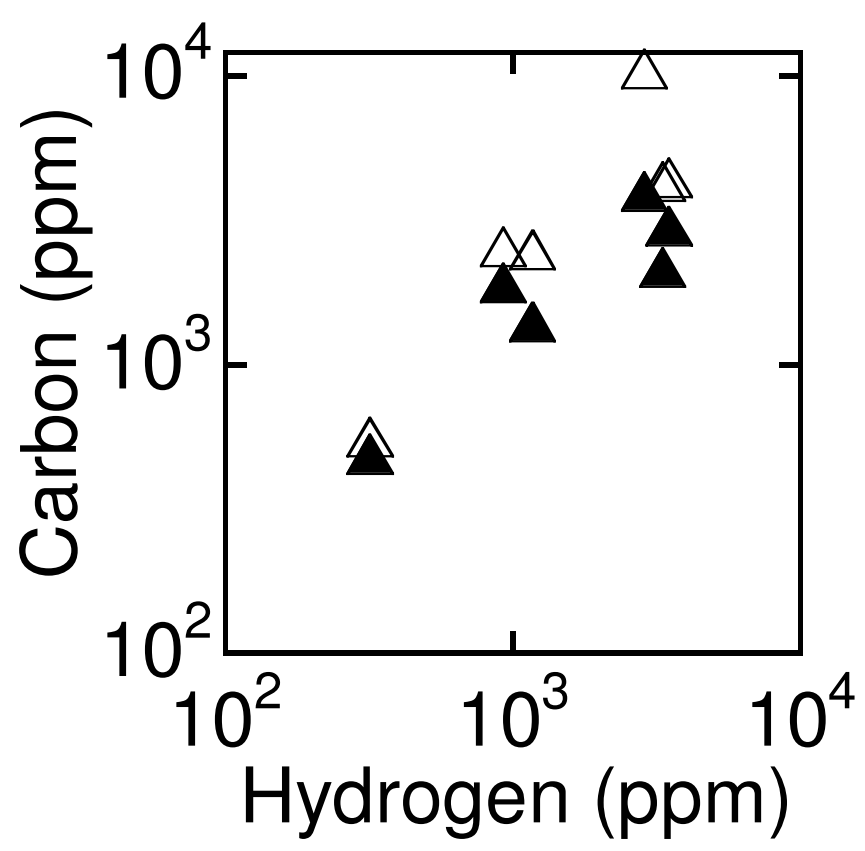

FIG. 2.-Relationship between the concentrations of surface-correlated C and $\mathrm{H}$ in different grains from lunar regolith 79035 . Refer to notes of Table 1 for details regarding calculations of the component concentrations. 
ionization potentials (or first ionization times) correspond well with their photospheric abundance ratios (Geiss, Gloeckler, \& von Steiger 1994).

\section{DISCUSSION}

The present experiment strongly suggests that solar carbon $\left(\delta^{13} \mathrm{C} \leq-105 \%\right.$ o $\left.\pm 20 \%\right)$ is depleted in ${ }^{13} \mathrm{C}$ by about $10 \%$ relative to meteoritic and planetary C (Fig. 3). Previous estimates of the solar isotopic composition (or analogues of the solar composition), e.g., the solar photosphere $(-74 \%$ o \pm $106 \%$; Hall 1973), the Jovian atmosphere $(-39 \%$ $\pm 46 \%$; Niemann et al. 1996), and comets $(-120 \%$ o $\pm 150 \%$; Wyckoff et al. 2000), are compatible with our estimate despite their large uncertainties. The $\delta^{13} \mathrm{C}$ values of bulk primitive meteorites (Robert \& Epstein 1982; Grady et al. 1986) between -30\%o and $0 \%$ are within the range of values observed for aromatic organic matter (Sephton \& Gilmour 2000), the main carrier of carbon in primitive meteorites. The planetary components found in the mantles of Earth $\left(\delta^{13} \mathrm{C} \sim 5 \%\right.$ ) and Mars $(-7 \%$ ) (Wright \& Pillinger 1994) are within this meteoritic range, suggesting that organic matter in primitive meteorites was a major contributor of carbon to planets and meteorites. Hereafter, we discuss the possible fractionation process that is responsible for the ${ }^{13} \mathrm{C}$ enrichment of organic compounds identified in primitive meteorites. Formation processes of meteoritic organics are not yet clearly understood and probably involve several steps of interstellar/circumstellar gas chemistry and processing in asteroidal parent bodies. However, by analogy to the case of $\mathrm{D}$ enrichment, which is widely accepted to be due to ion-molecule reactions in a low-temperature gas (Tielens 1997), the basic isotopic signatures commonly observed among the organics in the most primitive meteorites were probably acquired during the open-system processes that occurred in a gas medium (Sephton \& Gilmour 2000).

The first step of organic formation in space, i.e., conversion of $\mathrm{CO}$ to chemically more active forms of $\mathrm{C}$, is a major $\mathrm{C}$ isotopic fractionation process. Several mechanisms have been proposed: (1) an isotope exchange reaction between $\mathrm{CO}$ and C-bearing ions: ${ }^{12} \mathrm{CO}+{ }^{13} \mathrm{C}^{+} \rightarrow{ }^{13} \mathrm{CO}+{ }^{12} \mathrm{C}^{+}$, which is a slightly exothermic reaction $(\Delta E / k=35 \mathrm{~K})$ and is mostly efficient at a very low temperature of about $10 \mathrm{~K}$ (Langer \& Graedel 1989); (2) isotope-selective photodissociation of $\mathrm{CO}$ by ultraviolet (UV) light (van Dishoeck \& Black 1988; Warin et al. 1996); or (3) catalytic conversion of CO to hydrocarbon in a warm nebula by the so-called Fischer-Tropsch reaction (Kress \& Tielens 2001). Processes 1 and 3 tend to enrich the organic products in ${ }^{12} \mathrm{C}$ (Langer \& Graedel 1989; Yuen et al. 1990), whereas process 2 may cause a systematic enrichment of ${ }^{13} \mathrm{C}$ in the organics. In fact, the photodissociation rate of ${ }^{13} \mathrm{CO}$ by UV in a dense molecular cloud can be larger than that of ${ }^{12} \mathrm{CO}$, as a result of self-shielding of UV with wavelengths characteristic of ${ }^{12} \mathrm{CO}$ photodissociation. Astrochemical models (van Dishoeck \& Black 1988; Warin et al. 1996) consider that processes 1 and 2 play counter-roles on carbon isotopic fractionation, and they predict that an enrichment in ${ }^{13} \mathrm{C}$ of organics (i.e., ${ }^{12} \mathrm{C}$ enrichment of $\mathrm{CO}$ ) may occur in a dense and warm gas medium, where self-shielding of ${ }^{12} \mathrm{CO}$ lines is effective and isotopic fractionation by ion-molecule reactions is less efficient. However, in models simulating realistic molecular cloud conditions, delicate parameter tuning is required for production of a ${ }^{13} \mathrm{C}$ enrichment in organics. For example, in a cold $(10 \mathrm{~K})$ and dense $\left(10^{5} \mathrm{~cm}^{-3}\right)$ molecular cloud, organics would be slightly enriched in ${ }^{13} \mathrm{C}$, whereas

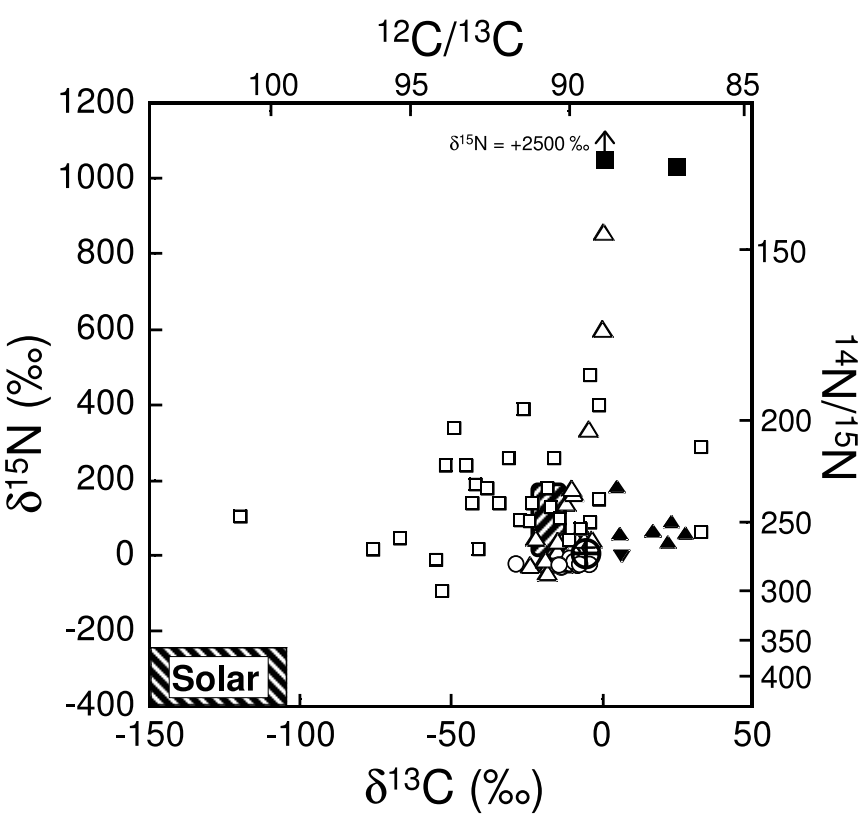

FIG. 3.-Comparison of $\mathrm{C}$ and $\mathrm{N}$ isotopic compositions among various planetary materials with the solar compositions. Whole-rock data of carbonaceous (Robert \& Epstein 1982; Kerridge 1985) and enstatite chondrites (Grady et al. 1986) are plotted as open triangles and circles, respectively. Data for interplanetary dust particles (Messenger 2000) are plotted as open squares. The solid squares represent the inferred end members in CR clan chondrites: $N \beta$ in the Bencubbin meteorite (Franchi, Wright, \& Pillinger 1986) and carbon silicate aggregates in CH chondrites (Sugiura \& Zashu 2001). Solid triangles and inverted triangles, respectively, represent data for the amino and carboxylic acids extracted from the Murchison meteorite (Epstein et al. 1987; Engel \& Macko 2001). The shaded area represents the $\mathrm{C}$ and $\mathrm{N}$ isotopic range for the aromatic (macromolecular) organics extracted from carbonaceous chondrites (Sephton \& Gilmour 2000; Alexander et al. 1998). The terrestrial mantle value is plotted with the Earth's symbol. The Martian mantle value overlaps with the terrestrial one within the size of the symbol. The upper limit of the solar $\delta^{15} \mathrm{~N}$ value is obtained from the ion probe measurement of the lunar regolith 79035 (Hashizume et al. 2000).

they would instead be enriched in ${ }^{12} \mathrm{C}$ when the density is $10^{4} \mathrm{~cm}^{-3}$ (Warin et al. 1996).

The ${ }^{13} \mathrm{C}$ enrichments could take place around $\mathrm{T}$ Tauri stars, where a warm $(\sim 100 \mathrm{~K}) \mathrm{H}_{2}$ gas has recently been detected (Thi et al. 2001). Such gas has been found to be enriched in radicals (e.g., $\mathrm{CN}$ ) that are effectively produced by photochemical reactions (Dutrey, Guilloteau, \& Guélin 1997). Recent protoplanetary disk models predict the existence of a warm, CO-rich molecular layer at the surface of the outer region of the disk irradiated by stellar UV (Aikawa et al. 2002), which could be a potential environment for synthesizing ${ }^{13} \mathrm{C}$-enriched organics. Such a possibility has to be compatible with the productions of the high $\mathrm{D} / \mathrm{H}$ and ${ }^{15} \mathrm{~N} /{ }^{14} \mathrm{~N}$ relative to solar that characterize meteoritic organics. For hydrogen, it has been suggested that D enrichment could have occurred via ion-molecule reactions in the protoplanetary disk, as well as in the molecular cloud (Aikawa \& Herbst 1999). For nitrogen, a couple of mechanisms have been proposed for ${ }^{15} \mathrm{~N}$ enhancement that could have prevailed in the protoplanetary disk. They are the self-shielding mechanism, similar to the case of $\mathrm{CO}$ that involves photochemistry of $\mathrm{N}_{2}$ (Clayton 2002), and ion-molecule reactions at the dense and cool $(\sim 10 \mathrm{~K})$ midplane of the outer disk, where most of the $\mathrm{CO}$ molecules (which hamper $\mathrm{N}$ isotopic fractionation) condense on grains 
and are removed from the gas phase (Charnley \& Rodgers 2002). The latter model has been proposed following the study of Terzieva \& Herbst (2000), which suggests that organics produced in (CO-rich) interstellar clouds could be enriched in ${ }^{15} \mathrm{~N}$ owing to ion-molecule exchange reactions; however, the predicted enrichment factor is at most $\sim 25 \%$ and may not account for the factor of 2 enhancement of the ${ }^{15} \mathrm{~N} /{ }^{14} \mathrm{~N}$ ratio of planetary $\mathrm{N}$ compared to protosolar $\mathrm{N}$ (Fig. 3).

Clayton (2002) recently proposed that isotopic fractionation could occur by the self-shielding mechanism involving photodissociation of $\mathrm{CO}$ and $\mathrm{N}_{2}$ in the near-solar region. In his model, the photodissociated atoms are rapidly trapped into refractory minerals (oxides, carbides, and nitrides) that are returned back to the cooler planet-forming region by the $\mathrm{X}$-wind process (Shu et al. 1997). Such processes have to be carefully modeled to test whether they can finally meet the observation that $\mathrm{C}$ and $\mathrm{N}$ in primitive meteorites are present predominantly in form of organics (Sephton \& Gilmour 2000; Alexander et al. 1998), i.e., find a way to transfer $\mathrm{C}$ and $\mathrm{N}$ from refractory phases to more fragile organics.

\section{SUMMARY AND CONCLUSIONS}

Two surface-correlated carbon components, whose abundances are correlated with those of solar hydrogen, were identified in lunar regolith sample 79035. These two components correspond most likely to the solar wind (SW) and the solar energetic particles (SEPs) implanted into lunar grains, which have contrasting $\mathrm{C}$ isotopic compositions $\left(\delta^{13} \mathrm{C} \leq\right.$ $-105 \%$ o $\pm 20 \%$ for SW and as high as $+10 \%$ for SEPs), the former likely representing the bulk solar and protosolar composition. Compared to the protosolar $\mathrm{C}$ isotopic composition, meteoritic organic $\mathrm{C}$ shows a systematic enrichment in ${ }^{13} \mathrm{C}$ of typically $10 \%$. It is possible that $\mathrm{C}$ isotopic fractionation occurred at the onset of organic formation in a CO-rich gas medium. Organic formation triggered by photodissociation of $\mathrm{CO}$ in a warm and dense gas medium is the most favorable process to explain the ${ }^{13} \mathrm{C}$ enrichment in the organics.

Samples were provided by NASA. This study was supported by the Mitsubishi Foundation, JSPS, and Region Lorraine. This is CRPG contribution 1644.
Aikawa, Y., \& Herbst, E. 1999, ApJ, 526, 314

Aikawa, Y., van Zadelhoff, G. J., van Dishoeck, E. F., \& Herbst, E. 2002, A\&A, 386, 622

Alexander, C. M. O., Russell, S. S., Arden, J. W., Ash, R. D., Grady, M. M., \& Pillinger, C. T. 1998, Meteoritics Planet. Sci., 33, 603

Anders, E., \& Grevesse, N. 1989, Geochim. Cosmochim. Acta, 53, 197

Becker, R. H. 1980, Earth Planet. Sci. Lett., 50, 189

Becker, R. H., \& Epstein, S. 1981, Lunar Planet. Sci. Conf., 12, 289

Bochsler, P. 1989, J. Geophys. Res., 94, 2365

Bustin, R., \& Gibson, E. K., Jr. 1992, The Second Conference on Lunar Bases and Space Activities of the 21 st Century, ed. W. W. Mendell (Houston: LPI), 437

Charnley, S. B., \& Rodgers, S. D. 2002, ApJ, 569, L133

Clayton, R. N. 2002, Nature, 415, 860

Dutrey, A., Guilloteau, S., \& Guélin, M. 1997, A\&A, 317, L55

Engel, M. H., \& Macko, S. A. 2001, Precambrian Res., 106, 35

Epstein, S., Krishnamurthy, R. V., Cronin, J. R., Pizzarello, S., \& Yuen, G. U. 1987, Nature, 326, 477

Franchi, I. A., Wright, I. P., \& Pillinger, C. T. 1986, Nature, 323, 138

Geiss, J., Gloeckler, G., \& von Steiger, R. 1994, Philos. Trans. R. Soc. London, A, 349, 213

Grady, M. M., Wright, I. P., Carr, L. P., \& Pillinger, C. T. 1986, Geochim. Cosmochim. Acta, 50, 2799

Hall, D. N. B. 1973, ApJ, 182, 977

Hashizume, K., Chaussidon, M., Marty, B., \& Robert, F. 2000, Science, 290,1142

Hashizume, K., Marty, B., \& Wieler, R. 2002, Earth Planet. Sci. Lett., 202, 201

Kerridge, J. F. 1985, Geochim. Cosmochim. Acta, 49, 1707

Kress, M. E., \& Tielens, A. G. G. M. 2001, Meteoritics Planet. Sci., 36, 75

Langer, W. D., \& Graedel, T. E. 1989, ApJS, 69, 241
REFERENCES

Li, Y. T., \& Wittenberg, L. J. 1992, The Second Conference on Lunar Bases and Space Activities of the 21st Century, ed. W. W. Mendell (Houston: LPI), 609

Messenger, S. 2000, Nature, 404, 968

Niemann, H. B., et al. 1996, Science, 272, 846

Owen, T., Mahaffy, P. R., Niemann, H. B., Atreya, S., \& Wong, M. 2001, ApJ, 553, L77

Robert, F., \& Epstein, S. 1982, Geochim. Cosmochim. Acta, 46, 81

Robert, F., Javoy, M., Halbout, J., Dimon, B., \& Merlivat, L. 1987, Geochim. Cosmochim. Acta, 51, 1787

Sephton, M. A., \& Gilmour I. 2000, ApJ, 540, 588

Shu, F. H., Shang, H., Glassgold, A. E., \& Lee, T. 1997, Science, 277, 1475

Smith, D., \& Adams, N. G. 1980, ApJ, 242, 424

Sugiura, N., \& Zashu, S. 2001, Meteoritics Planet. Sci., 36, 515

Terzieva, R., \& Herbst, E. 2000, MNRAS, 317, 563

Thi, W. F., et al. 2001, ApJ, 561, 1074

Tielens, A. G. G. M. 1997, in AIP Conf. Proc. 402, Astrophysical Implications of the Laboratory Study of Presolar Materials, ed. T. J. Bernatowicz \& E. K. Zinner (New York: AIP), 523

van Dishoeck, E. F., \& Black, J. H. 1988, ApJ, 334, 771

Warin, S., Benayoun, J. J., \& Viala, Y. P. 1996, A\&A, 308, 535

Wieler, R. 1998, Space Sci. Rev., 85, 303

Wieler, R., Kehm, K., Meshik, A. P., \& Hohenberg, C. M. 1996, Nature, 384,46

Wright, I. P., \& Pillinger, C. T. 1994, Philos. Trans. R. Soc. London A, 349, 309

Wyckoff, S., Kleine, M., Peterson, B. A., Wehinger, P. A., \& Ziurys, L. M. 2000, ApJ, 535, 991

Yuen, G. U., et al. 1990, Lunar Planet. Sci. Conf., 21, 1367

Ziegler, J. F., Biersack, J. P., \& Littmark, U. 1985, The Stopping and Ranges of Ions in Matter, Vol. 1 (New York: Pergamon) 